\title{
The tumor suppressor miR-124 inhibits cell proliferation by targeting STAT3 and functions as a prognostic marker for postoperative NSCLC patients
}

\author{
XIUMEI LI ${ }^{1 *}$, ZHUANG YU ${ }^{1 *}$, YONG LI ${ }^{1}$, SHIHAI LIU ${ }^{2}$, \\ CAIHONG GAO $^{1}$, XIN HOU ${ }^{1}$, RUYONG YAO ${ }^{2}$ and LIANHUA CUI ${ }^{3}$ \\ Departments of ${ }^{1}$ Oncology and ${ }^{2}$ Central Laboratory, The Affiliated Hospital of Qingdao University, \\ Qingdao, Shandong 266003; ${ }^{3}$ Department of Public Health, Qingdao University \\ Medical College, Qingdao, Shandong 266021, P.R. China
}

Received September 26, 2014; Accepted November 12, 2014

DOI: 10.3892/ijo.2014.2786

\begin{abstract}
The aim of the present study was to investigate the role of miR-124 in lung cancer and identify the potential predictive value of miR-124 in postoperative non-small cell lung cancer (NSCLC) patients. We detected miR-124 expression in A549, NCL-H460 and normal lung epithelial BEAS-2E cells and showed a significantly lower expression level of miR-124 in NSCLC cells than in BEAS-2E cells. Upregulation of miR-124 expression levels in both A549 and NCL-H460 cells by transfection with miR-124 mimics suppressed cell proliferation and induced apoptosis. Further investigation revealed that miR-124 bound directly to the 3 ' UTR region of STAT3, thereby inhibiting STAT3 expression. In addition, miR-124 levels detected in NSCLC tissues were lower than those in adjacent normal lung tissues, while the opposite was observed for STAT3. In NSCLC, the expression levels of miR-124 and STAT3 correlated significantly with the tumor node metastases (TNM) stage, differentiation grade and lymph node metastasis, while the levels of these molecules did not differ significantly by gender, age, location, smoking index, pleural invasion or pathological type. The expression level of miR-124 was significantly associated with diseasefree survival (DFS) in both positive and negative lymph node groups. Furthermore, patients with low miR-124 or high STAT3 expression generally received a worse prognosis in terms of both overall survival (OS) and DFS. In conclusion, our findings suggest that miR-124 functions as a tumor suppressor by targeting STAT3, and that miR-124 may potentially serve as a useful biomarker for the prognosis of NSCLC patients.
\end{abstract}

Correspondence to: Dr Zhuang Yu, Department of Oncology, The Affiliated Hospital of Qingdao University, 16 Jiangsu Road, Qingdao, Shandong 266003, P.R. China

E-mail: yczhmed@126.com

${ }^{*}$ Contributed equally

Key words: miR-124, prognosis, non-small cell lung cancer, STAT3

\section{Introduction}

Lung cancer is the leading cause of cancer-related mortality both globally and in China. Statistics indicate that 884,500 new lung cancer cases were diagnosed and 778,000 individuals died from lung cancer in developing countries in 2008 alone (1). Specifically, non-small cell lung cancer (NSCLC) accounts for $\sim 80-85 \%$ of lung cancer cases (2). However, the lack of effective early detection methods and limited options for combined therapies both contribute to the dismal statistics associated with lung cancer. Since the initial identification of the lethal-7 (let-7) gene as a regulator in Caenorhabditis elegans development, members of the let-7 family have frequently been deleted in lung cancer, cell lines, mouse NSCLC xenografts and NSCLC patients (3-6). Subsequent studies have also identified additional microRNAs (miRNAs) and implicated several dysregulated miRNAs in lung cancer.

miRNAs are short endogenous non-protein-coding RNAs that are 22 nucleotides in length and play important roles in regulating human gene expression. By base pairing to the $3^{\prime}$ untranslated region ( $3^{\prime}$ UTR) of the target messenger RNA (mRNA), miRNAs mediate post-transcriptional gene silencing by inhibiting mRNA translation or cleaving the target mRNA $(7,8)$. Various miRNAs are involved in diverse cellular processes, including cell proliferation, apoptosis, development and differentiation $(6,9)$. Accumulating evidence shows that miRNAs function as oncogenes or tumor suppressors and can be grossly dysregulated in various cancers including lung cancer (10).

miR-124, a brain-specific miRNA, was first reported to be abundantly expressed in neuronal cells (11). Recent studies have suggested that miR-124 is significantly downregulated in several human cancers, such as gastric, cervical, bladder and breast cancer. These studies also suggest that this downregulation may be caused by DNA hypermethylation (12-14). In addition, several studies have shown that miR-124 exhibits oncogenic activity by directly suppressing signal transducer and activator of transcription 3 (STAT3) expression $(15,16)$. STAT3, which might be an important target of miR-124, upregulates the expression of cyclin D1, survivin, Bcl-xL 
and vascular endothelial growth factor (VEGF), which are responsible for suppressing cell apoptosis and inducing tumor growth (17-20). To date, increasing evidence has indicated that miR-124 may function as a tumor suppressive factor by downregulating STAT3 in various human cancers. However, the relationship between miR-124 function and lung cancer remains unclear.

In the present study, we explored the role of miR-124 in lung cancer and searched for a possible relationship between miR-124 and STAT3 using TargetScan software. The findings were confirmed using a luciferase assay. In addition, we retrospectively examined the expression of miR-124 and its target gene STAT3 in postoperative NSCLC samples and adjacent normal lung tissues. Furthermore, we investigated the relationship between miR-124 and clinicopathological features and survival in NSCLC patients, the results of which suggested that miR-124 might be a clinically useful biomarker for the prognosis of NSCLC.

\section{Materials and methods}

Patients and tissue specimens. All of the patients included in the study underwent surgical resection procedures at the Thoracic Surgery Department of the Affiliated Hospital of Qingdao University, Qingdao, China. One hundred and sixty-four eligible patients diagnosed with a primary tumor in stages I, II or III were enrolled into the present study between February 2009 and May 2013. None of the patients had received any treatment for lung cancer prior to surgery, and all patients had a preoperative computed tomography scan. Patients with diffuse lobar or multifocal bronchioalveolar carcinoma and patients who had malignancy within the previous 5 years were ineligible. The NSCLC stage was based on the American Joint Committee on Cancer (AJCC) TNM System. Tumors were graded as well, moderately or poorly differentiated. After the surgical specimens were obtained, individual-matched normal lung tissues adjacent to the proximal excision margin were harvested. A total of 164 pairs of specimens were used to generate paraffin-embedded tissue microarrays. The remaining specimens were immediately snap-frozen in liquid nitrogen and stored at $-80^{\circ} \mathrm{C}$ until further analysis.

Cell culture. The A549 human lung cancer cell line, NCL-H460 cells and BEAS-2E normal lung epithelial cells were obtained from the Cell Resource Center of Shanghai Institutes for Biological Sciences. The cell lines were routinely cultured in DMEM-1640 medium (Gibco, Carlsbad, CA, USA) supplemented with $10 \%$ fetal bovine serum (FBS; Gibco), $100 \mathrm{U} / \mathrm{ml}$ penicillin-G, and $100 \mu \mathrm{g} / \mathrm{ml}$ streptomycin, and were cultivated in a humidified incubator with $5 \% \mathrm{CO}_{2}$ at $37^{\circ} \mathrm{C}$.

Cell transfection. The miR-124 mimics (miR-124) and negative control mimics (miR-NC) were all purchased from Beyotime Institute of Biotechnology (Co., Ltd., Shanghai, China). The cells were seeded in 12-well plates and grown to $40 \%$ confluence before transfection. The oligonucleotides were transfected at a final concentration of $50 \mathrm{nM}$ using Lipofectamine 2000 (Invitrogen, Carlsbad, CA, USA) in accordance with the manufacturer's instructions. The cells were divided into three groups according to the treatment and were named as follows: i) miR-124 (transfected with miR-124mimics); ii) miR-NC (transfected with negative control mimics); and iii) untransfected control. RNA or protein was isolated $48 \mathrm{~h}$ after transfection and used for qRT-PCR or western blotting, respectively. The transfections were repeated in triplicate a minimum of 3 times.

Cell viability analysis. Cell proliferation was determined using methyl thiazolyl tetrazolium (MTT) assays. Briefly, A549 cells $\left(5 \times 10^{3}\right.$ cells/well) were plated in 96-well plates with DMEM containing $10 \%$ FBS. After $24 \mathrm{~h}$ of culturing, the A549 cells were transfected with miR-124 or miR-NC mimics as described above. The MTT assay was completed 0, 12, 24, 48 and $72 \mathrm{~h}$ after gene transfection. The absorbance in each well was measured with a microplate reader set at 450 and $630 \mathrm{nM}$. All experiments were performed in triplicate and repeated three times. For the apoptosis analysis, cultured cells were harvested by trypsinization and washed twice with ice-cold PBS. Forty-eight hours after transfection, the cells $\left(5 \times 10^{5}\right.$ cells/ $\mathrm{ml}$ ) from each sample were stained with Annexin V-FITC and PI using the protocol included in the Apoptosis Detection kit I (BD Biosciences, San Jose, CA, USA). A fluorescence-activated cell sorting FACSCalibur instrument (BD Biosciences) was used for this assay, and the data generated were subsequently analyzed with CellQuest software (BD Biosciences). The experiments were performed in triplicate and repeated three times.

Luciferase assay. The 3' UTR of STAT3 containing wild-type or mutant miR-124 target sites was amplified by PCR and cloned into the XhoI/NotI site of the pmiR-RB-REPORT vector. The XhoI/NotI site was downstream of the Renilla reporter gene. The mutant constructs were created by mutating the seed regions of the miR-124 sites (5'-GUGCCU-3'). The primers for the wild-type STAT3 3' UTR were 5'-CCCTCGA GGGAGCTGAGAACGGAAGCTGCA-3' (forward) and 5'-ATTTGCGGCCGCAGCTGTTCTGCCTCACCTGTG GG-3' (reverse), while the mutagenesis primers were 5'-CC CTGATATCACATCCACAGAAACAACCT-3' (reverse) and 5'-AGGTTGT TTCTGTGGATGTGATATCAGGG-3' (forward). All of the constructs were confirmed using restriction digests and DNA sequencing. For the luciferase reporter assay, the A549 cell line was co-transfected with luciferase constructs containing the 3' UTR of STAT3 (with wild-type or mutant miR-124 binding sites) and miR-124 or miR-NC mimics. All of the transfections were conducted in triplicate with Lipofectamine 2000. Luciferase activity was measured $48 \mathrm{~h}$ after transfection using a dual luciferase reporter system (Promega, Madison, WI, USA) according to the manufacturer's protocol and normalized to the corresponding Renilla luciferase activity.

Quantitative real-time reverse transcription polymerase chain reaction ( $q R T-P C R)$. Total RNA was extracted from fresh tissues or cells using TRIzol reagent (Gibco). The concentration and purity of the RNA was determined using a NanoDrop ${ }^{\circledR}$ ND-1000 spectrophotometer (Thermo Fisher Scientific, Inc., Wilmington, DE, USA). miR-124 expression was determined using a TaqMan microRNA assay kit (Applied Biosystems, Foster City, CA, USA). To determine the expression of STAT3 
Table I. A list of PCR primers used in the reactions.

\begin{tabular}{ll}
\hline Gene & \multicolumn{1}{c}{ Primer sequences } \\
\hline miR-124 & F: 5'-GGACTTTCTTCATTCACACCG-3' \\
& R: 5'-GACCACTGAGGTTAGAGCCA-3' \\
U6 & F: 5'-CTCGCTTCGGCAGCACATATACT-3' \\
& R: 5'-ACGCTTCACGAATTTGCGTGTC-3' \\
STAT3 & F: 5'-GAAGAATCCAACAACGGC-3' \\
& R: 5'-TCACAATCAGGGAAGCAT-3' \\
GAPDH & F: 5'-CAACGACCACTTTGTCAAGCTCA-3' \\
& R: 5'-GCTGGTGGTCCAGGGGTCTTACT-3' \\
\hline
\end{tabular}

STAT3, signal transducer and activator of transcription 3; GAPDH, glyceraldehyde-3-phosphate dehydrogenase. F, forward; R, reverse.

and glyceraldehyde-3-phosphate dehydrogenase (GAPDH), $1 \mu \mathrm{g}$ of total RNA was subjected to first-strand cDNA synthesis (Takara, Shiga, Japan) and examined using a PrimeScript RT reagent kit (Takara). Real-time PCR was performed using SYBR-Green PCR Master Mix (Takara) on the ABI 7500HT System. All of the primers used in the present study were designed by Sangon Biotech Co., Ltd. (Shanghai, China). The expression of U6 and GAPDH was used for normalization. The relative expression of mRNA was determined using the $2^{-\Delta \Delta C t}$ method (21). All of the real-time PCR reactions were carried out in triplicate. A list of primers used in the reactions is presented in Table I.

Western blotting. Tissues were lysed using RIPA buffer (Sigma, St. Louis, MO, USA). The soluble protein concentration was determined by the Bradford method. Total protein $(10 \mu \mathrm{g})$ was run on a $10-15 \%$ SDS-PAGE gel and transferred to polyvinylidene difluoride (PVDF) membranes. The membranes were blocked with $1 \%$ Tween 20 -PBS at $4^{\circ} \mathrm{C}$ overnight before being washed and incubated with primary antibodies against STAT3 and GAPDH (Bioworld Co., Dublin, OH, USA; diluted 1:500 in TBS-A) for $1 \mathrm{~h}$ at room temperature. After washing, the membranes were incubated with the secondary antibodies (Abcam Co., Cambridge, UK; diluted 1:1,000 in TBS-A) for $1 \mathrm{~h}$ at room temperature. The immunoblots were developed using an electrochemiluminescence kit and imaged with the Vilber Fusion FX5 automatic gel imaging analysis system (Vilber, Marne La Vallée, France).

Immunohistochemistry (IHC). Immunohistochemistry was performed using standard techniques. Briefly, 4- $\mu \mathrm{m}$ thick paraffin-embedded specimens were dewaxed in xylene and rehydrated in graded alcohols. Endogenous peroxidase activity was blocked using 3\% hydrogen peroxide. Antigen retrieval was accomplished in citrate buffer ( $\mathrm{pH}$ 6.0) using a microwave. A polyclonal rabbit anti-human STAT3 antibody (1:200 dilution; Santa Cruz Biotechnology, Santa Cruz, CA, USA) was added and the samples were incubated overnight at $4^{\circ} \mathrm{C}$. After washing, the sections were incubated with a secondary antibody for $40 \mathrm{~min}$ at $37^{\circ} \mathrm{C}$ and subsequently incubated with streptavidin-conjugated horseradish peroxidase (HRP) and diaminobenzidine (DAB) and counterstained with hematoxylin. The primary antibody was replaced by an isotope IgG in the negative control. Representative viable tissue sections were scored semi-quantitatively using light microscopy. The proportion of positively stained cells was graded as follows: score 0 , completely negative samples; score 1 , samples with up to $10 \%$ positive cells; score 2 , samples with $11-50 \%$ positive cells; and score 3 , samples with $50 \%$ positive cells. The staining intensity in tumor cells was scored as 0 , no staining, 1 , weak staining, 2 , moderate staining or 3 , strong staining. The staining index was calculated as the product of the staining intensity score and the proportion score, which had a value of $0,1,2,3,4,6$ or 9 . The final score was achieved by comparing the scores obtained by different observers, and any discrepancies were resolved by consensus. In the case of a disagreement, the slides were reexamined and the observers reached a consensus. A staining index $\geq 4$ was defined as high expression, and an index $<4$ was defined as low expression.

Follow-up. Follow-up was carried out by telephone or written correspondence, while clinical examinations and chest radiography were performed every 3 months for 3 years and every 6 months thereafter. Disease-free survival (DFS) was evaluated for the period from the date of operation to the appearance of tumor progression. Overall survival (OS) was evaluated for the period from the date of surgery to the date of death (or last follow-up). The study concluded on December 31, 2013. The overall follow-up rate was $100 \%$, and the median follow-up time was 31 months with a range from 2 months to 58 months.

Ethics statement. All of the specimens were obtained from the Central Laboratory of the Affiliated Hospital of Qingdao University, and all of the patients provided written informed consent for the use of their tissues. The present study was approved by the Ethics Committee of the Affiliated Hospital of Qingdao University.

Statistical analysis. All of the analyses were performed using SPSS 19.0 software (SPSS, Inc., Chicago, IL, USA) and GraphPad Prism 5.0 (GraphPad Software, San Diego, CA, USA). All of the numerical data were expressed as the mean \pm SD. P-values $<0.05$ were defined as statistically significant. The results were analyzed using the Student's t-test or one-way analysis of variance (ANOVA). The Chi-square test was used to analyze the relationship between miR-124 or STAT3 expression and clinicopathological symptoms. Bivariate correlations between the study variables were calculated using Spearman's rank-order correlation coefficients. The survival curves were analyzed using Kaplan-Meier methodology, and the difference in distribution was evaluated with the log-rank test. The influence of each variable on survival was examined by univariate and multivariate Cox regression analysis.

\section{Results}

Patient characteristics. A total of 164 patients were enrolled in the present study (Table II). The patients consisted of 115 men and 49 women with a median age of 59 years (range, 22-76 years). The NSCLC tumors consisted of 106 (64.6\%) adeno- 
Table II. Clinicopathological features of patients and miR-124 and STAT3 expression levels in NSCLC.

\begin{tabular}{|c|c|c|c|c|c|c|c|}
\hline \multirow[b]{2}{*}{ Characteristics } & \multirow[b]{2}{*}{ Cases 164} & \multicolumn{2}{|c|}{ miR-124 expression level } & \multirow[b]{2}{*}{ P-value } & \multicolumn{2}{|c|}{ STAT3 expression level } & \multirow[b]{2}{*}{ P-value } \\
\hline & & $\begin{array}{c}\text { Low } \\
\text { expression } \\
\mathrm{n}=116(\%)\end{array}$ & $\begin{array}{c}\text { High } \\
\text { expression } \\
\mathrm{n}=48(\%)\end{array}$ & & $\begin{array}{c}\text { Low } \\
\text { expression } \\
\mathrm{n}=57(\%)\end{array}$ & $\begin{array}{c}\text { High } \\
\text { expression } \\
\mathrm{n}=107(\%)\end{array}$ & \\
\hline Gender & & & & 0.105 & & & 0.290 \\
\hline Male & 115 & 85 (81.34) & $30(33.66)$ & & $38(39.97)$ & $77(75.03)$ & \\
\hline Female & 49 & $31(34.66)$ & $18(14.34)$ & & $19(17.03)$ & $30(31.97)$ & \\
\hline Age (years) & & & & 0.378 & & & 0.316 \\
\hline$\geq 60$ & 75 & $50(53.05)$ & $25(21.95)$ & & $34(26.07)$ & $41(48.93)$ & \\
\hline$<60$ & 89 & $66(62.95)$ & $23(26.05)$ & & $23(30.93)$ & $66(58.07)$ & \\
\hline Smoking index (SI) & & & & 0.565 & & & 0.349 \\
\hline$\geq 400$ & 86 & $62(60.83)$ & $24(25.17)$ & & $28(29.89)$ & $58(56.11)$ & \\
\hline$<400$ & 78 & $54(55.17)$ & $24(22.83)$ & & $29(27.11)$ & $49(50.89)$ & \\
\hline Tumor site & & & & 0.208 & & & 0.436 \\
\hline Left lobe & 64 & $44(45.27)$ & $20(18.73)$ & & $25(22.24)$ & $39(41.76)$ & \\
\hline Right lobe & 100 & $72(70.73)$ & $28(29.27)$ & & $32(34.76)$ & $68(65.24)$ & \\
\hline Pleural invasion & & & & 0.190 & & & 0.815 \\
\hline Absent & 45 & $36(31.83)$ & $9(13.17)$ & & 15 (15.64) & $30(29.36)$ & \\
\hline Present & 119 & $80(84.17)$ & $39(34.83)$ & & $42(41.36)$ & $77(77.64)$ & \\
\hline Lymph node metastasis & & & & 0.017 & & & 0.008 \\
\hline Absent & 61 & $37(43.15)$ & $24(17.85)$ & & $30(21.20)$ & $31(39.80)$ & \\
\hline Present & 103 & $79(72.85)$ & $24(30.15)$ & & $27(35.80)$ & $76(67.20)$ & \\
\hline TNM stage & & & & 0.006 & & & 0.007 \\
\hline IA & 5 & $2(3.54)$ & $3(1.46)$ & & $4(1.74)$ & $1(3.26)$ & \\
\hline IB & 42 & $25(29.71)$ & $17(12.29)$ & & $19(14.60)$ & $23(27.40)$ & \\
\hline IIA & 22 & $15(15.56)$ & $7(6.44)$ & & $7(7.65)$ & $15(14.35)$ & \\
\hline IIB & 7 & $6(4.95)$ & $1(2.05)$ & & $4(2.43)$ & $3(4.57)$ & \\
\hline IIIA & 57 & $43(40.32)$ & $14(16.68)$ & & $16(19.81)$ & $41(37.19)$ & \\
\hline IIIB & 31 & $25(21.93)$ & $6(9.07)$ & & $7(10.77)$ & $24(20.23)$ & \\
\hline Differentiation & & & & 0.001 & & & $<0.001$ \\
\hline Well & 13 & $5(9.20)$ & $8(3.80)$ & & $9(4.52)$ & $4(8.48)$ & \\
\hline Moderately & 63 & $38(44.56)$ & $25(18.44)$ & & $33(21.90)$ & $30(41.10)$ & \\
\hline Poorly & 86 & $71(60.83)$ & $15(25.17)$ & & $15(29.89)$ & $71(56.11)$ & \\
\hline Pathological type & & & & 0.578 & & & 0.838 \\
\hline $\mathrm{AC}$ & 106 & $74(74.98)$ & $32(31.02)$ & & $37(36.84)$ & $70(69.16)$ & \\
\hline $\mathrm{SCC}$ & 52 & 37 (36.78) & $15(15.22)$ & & $18(18.07)$ & $34(33.93)$ & \\
\hline Other types & 6 & $5(4.24)$ & $1(1.76)$ & & $2(2.09)$ & $4(3.91)$ & \\
\hline
\end{tabular}

NSCLC, non-small cell lung cancer; AC, adenocarcinoma; SCC, squamous cell carcinoma.

carcinomas (AC), $52(31.7 \%)$ squamous cell carcinomas (SCC) and $6(3.7 \%)$ carcinomas of other cell types. The post-surgical pathologic stage was determined by the TNM classification. The number of patients with disease stages I, II and III was 47 (28.6\%), 29 (17.7\%) and $88(53.7 \%)$, respectively.

miR-124 is downregulated in human NSCLC cells. We performed real-time PCR in the A549 and NCL-H460 NSCLC cell lines and the BEAS-2E normal lung epithelial line to confirm the expression level of miR-124. The results showed that miR-124 expression in both A549 and NCL-H460 cells was significantly lower than in normal lung epithelial BEAS-2E cells ( $\mathrm{P}=0.0015$ and $\mathrm{P}<0.001$, respectively) (Fig. 1A), confirming the downregulation of miR-124 in NSCLC cells.

miR-124 inhibits cell proliferation and induces apoptosis in A549 cells. To explore the expression and significance of miR-124 in lung cancer, we transfected miR-124 mimics or negative control mimics into both A549 and NCL-H460 cells. The expression level of miR-124 was detected using qRT-PCR 

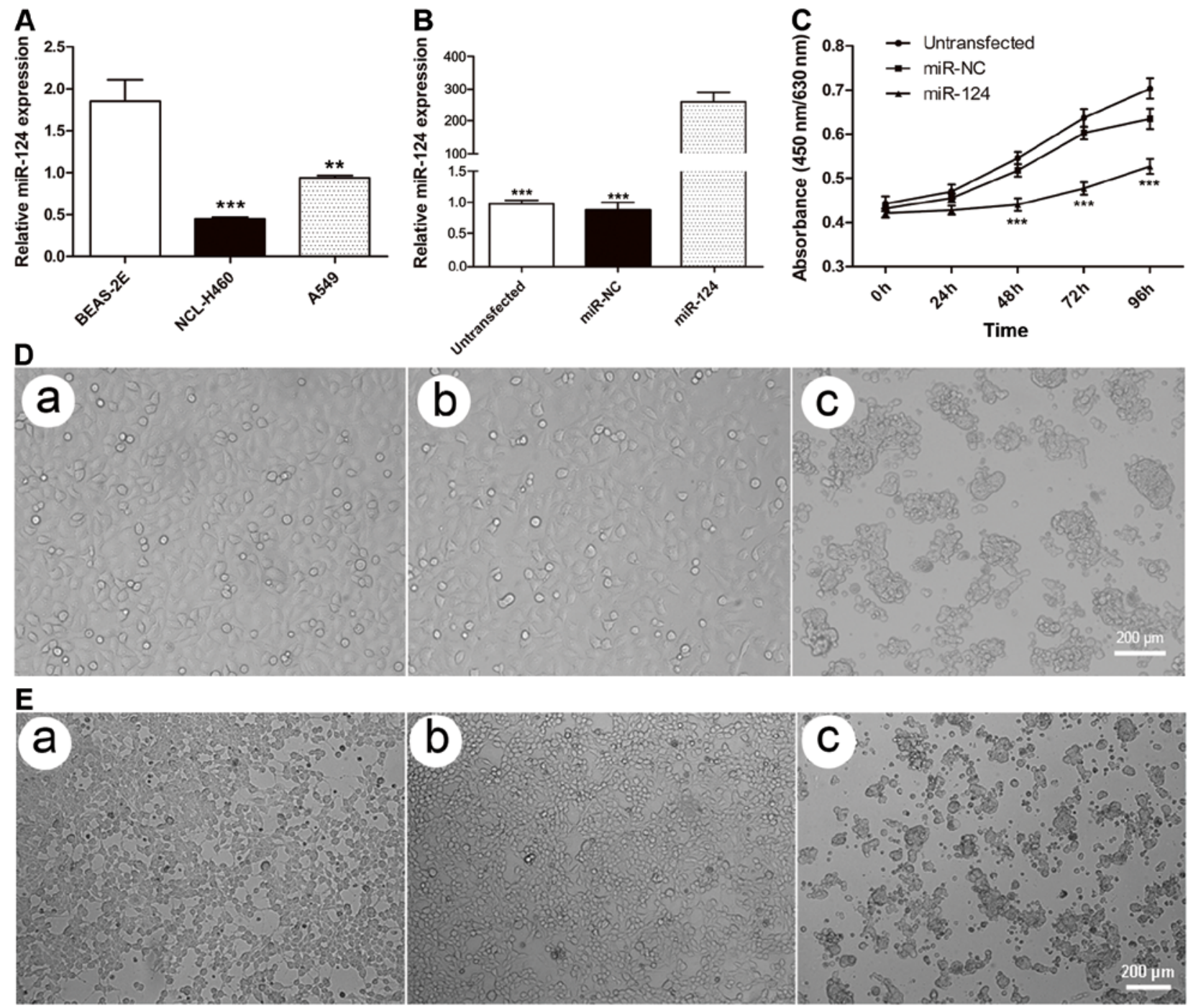

$\mathbf{F}$
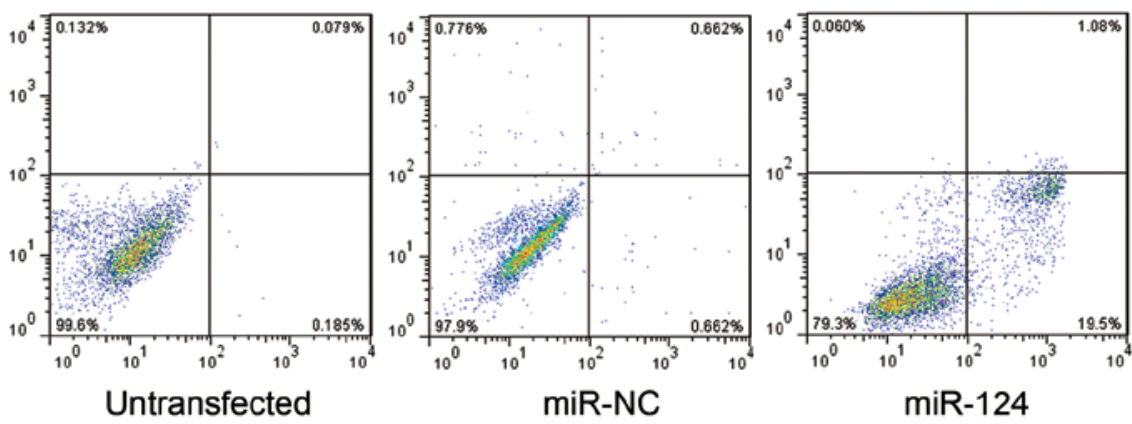

G

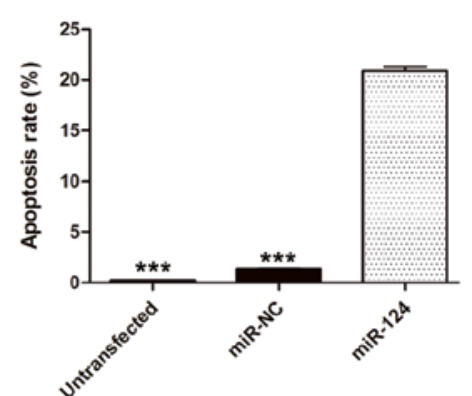

Figure 1. miR-124 expression in NSCLC cells and its effects on cell proliferation and apoptosis. (A) The expression level of miR-124 detected by qRT-PCR was significantly lower in A549 and NCL-H460 cells than in the BEAS-2E cell line ( $\mathrm{P}=0.0015$ vs. $\mathrm{P}<0.001)$. (B) QRT-PCR was used to detect the expression of miR-124 in NSCLC cell lines $48 \mathrm{~h}$ after transfection. The expression of miR-124 increased in both A549 and NCL-H460 cells transfected with miR-124 mimics. (C) Cell viability was examined using an MTT assay $96 \mathrm{~h}$ after transfection with miR-124 mimics or negative control mimics. The cell proliferation rate of cells transfected with miR-124 was suppressed compared with the other two cell lines. Morphology of (D) A549 and (E) NCL-H460 cell lines (magnification, x100): a) untransfected cells; b) miR-NC; and c) miR-124. Most of the cells died after successful transfection with miR-124 mimics, while the shape, volume and membrane were altered in surviving cells. (F) Apoptosis was determined by fluorescence-activated cell sorting (FACS) $48 \mathrm{~h}$ after transfection. Cells transfected with miR-124 that were in early apoptosis can be seen in the bottom right quadrant, while cells in late apoptosis are in the top right quadrant. (G) The apoptosis rate of the three cell lines is shown. NSCLC, non-small cell lung cancer; ${ }^{* *} \mathrm{P}<0.01 ;{ }^{* * *} \mathrm{P}<0.001$.

$48 \mathrm{~h}$ after transfection. The cells transfected with miR-124 mimics showed a significant, 300 -fold increase in miR-124 expression compared with miR-NC cells or untransfected cells $(\mathrm{P}<0.001)$ (Fig. 1B). We further investigated the effect of miR-124 on cell survival. The cell lines transfected with
miR-124 mimics were significantly less viable 24,48 and $72 \mathrm{~h}$ post-transfection compared with miR-NC cells or untransfected cells (Fig. 1C). The cells overexpressing miR-124 began to shrink in size and display vague membranes and an irregular shape $48 \mathrm{~h}$ after transfection (Fig. 1D and E). Few 

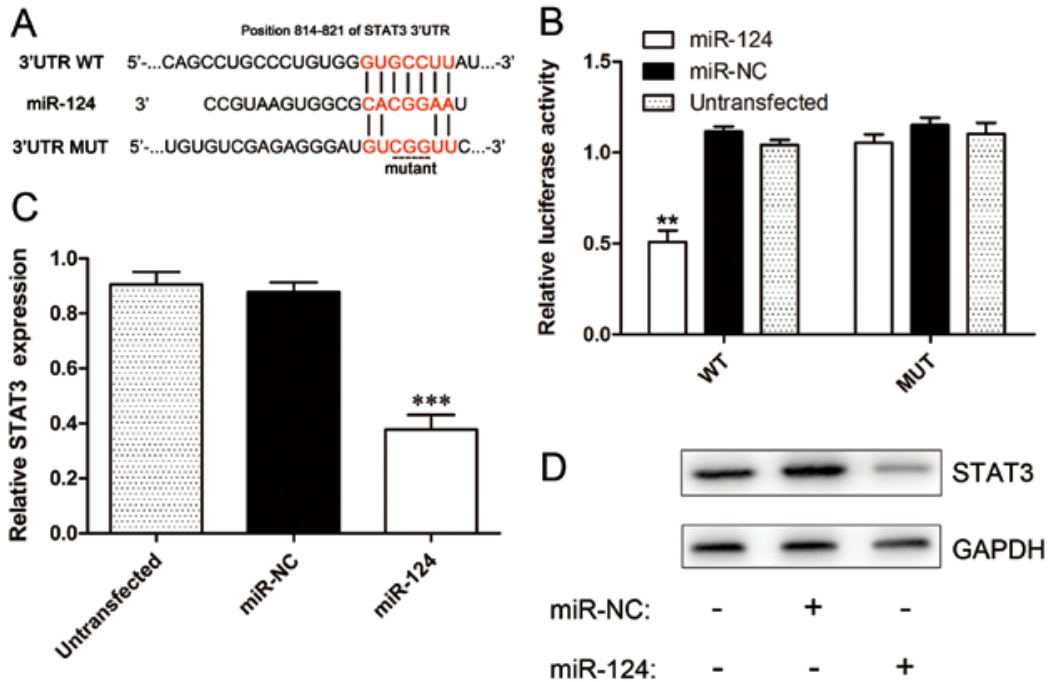

Figure 2. STAT3 is a direct target of miR-124. (A) The STAT3 3' UTR fragment containing wild-type or mutant miR-124 target sites is shown. (B) Compared with A549-miR-NC or untransfected cells, the luciferase activity of the wild-type STAT3 3' UTR construct was suppressed in A549-miR-124 cells (P=0.0024 vs. $\mathrm{P}=0.0032$, respectively). (C) The relative expression level of STAT3 mRNA in three cell lines was determined by real-time PCR $48 \mathrm{~h}$ after transfection. The expression of STAT3 in A549-miR-124 cells was significantly downregulated as compared with the other two cell lines. (D) Western blot analysis of STAT3 expression in cell lines after transfection of miR-124. WT, wild-type; MUT, mutant; STAT3, signal transducer and activator of transcription 3; 3' UTR, 3' untranslated region; $\mathrm{NC}$, negative control; ${ }^{* *} \mathrm{P}<0.01 ;{ }^{* * *} \mathrm{P}<0.001$.

A

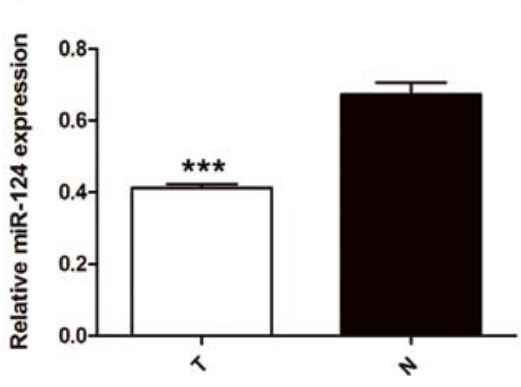

C
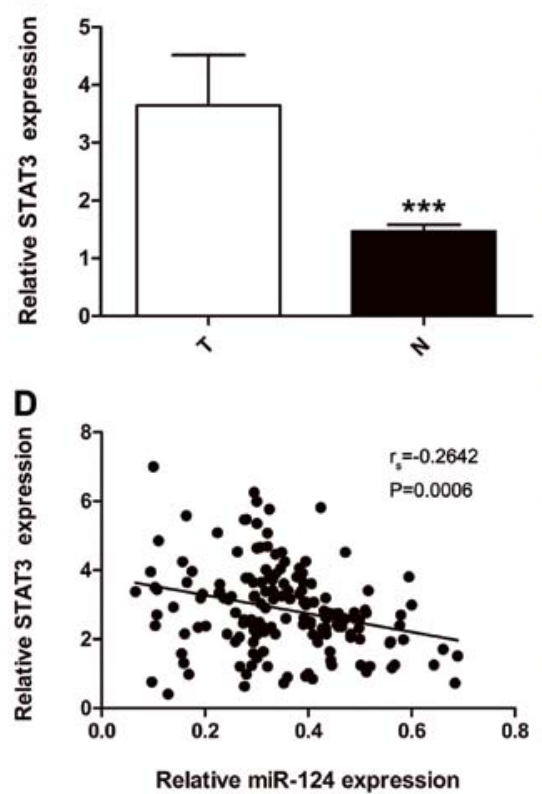
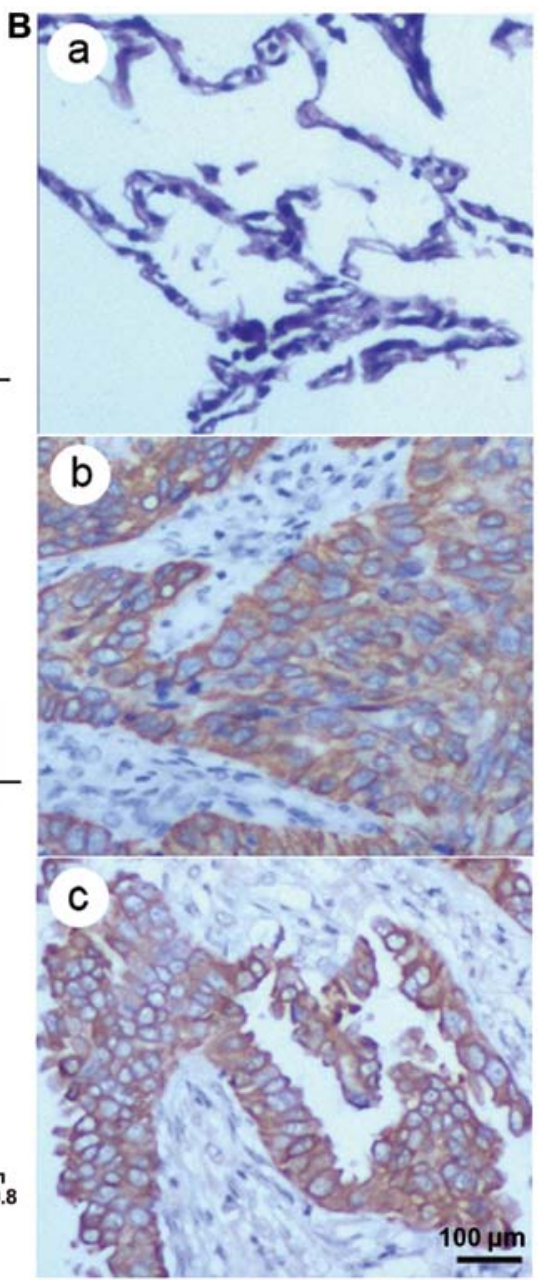

Figure 3. The expression of miR-124 and STAT3 in NSCLC tissues and adjacent normal lung tissues. (A) The relative expression of miR-124 mRNA was lower in NSCLC tissue than adjacent normal lung tissue. (B) Immunohistochemical staining for STAT3 in NSCLC tissue and adjacent normal lung tissue (magnification, x200). The STAT3 staining was primarily localized to the cytoplasm and rarely detected in the nucleus. a) Normal lung tissue; b) squamous cell carcinoma; c) adenocarcinoma. (C) The relative expression level of STAT3 was significantly increased in NSCLC tissues than adjacent normal lung tissues. (D) Spearman's rank-order correlation was used to show the inverse correlation between miR-124 and STAT3 expression in NSCLC tissues $\left(r_{s}=-0.2642\right.$, $\mathrm{P}=0.0006)$. $\mathrm{T}$, tumor tissue; $\mathrm{N}$, normal lung tissue; ${ }^{* * *} \mathrm{P}<0.001$. 

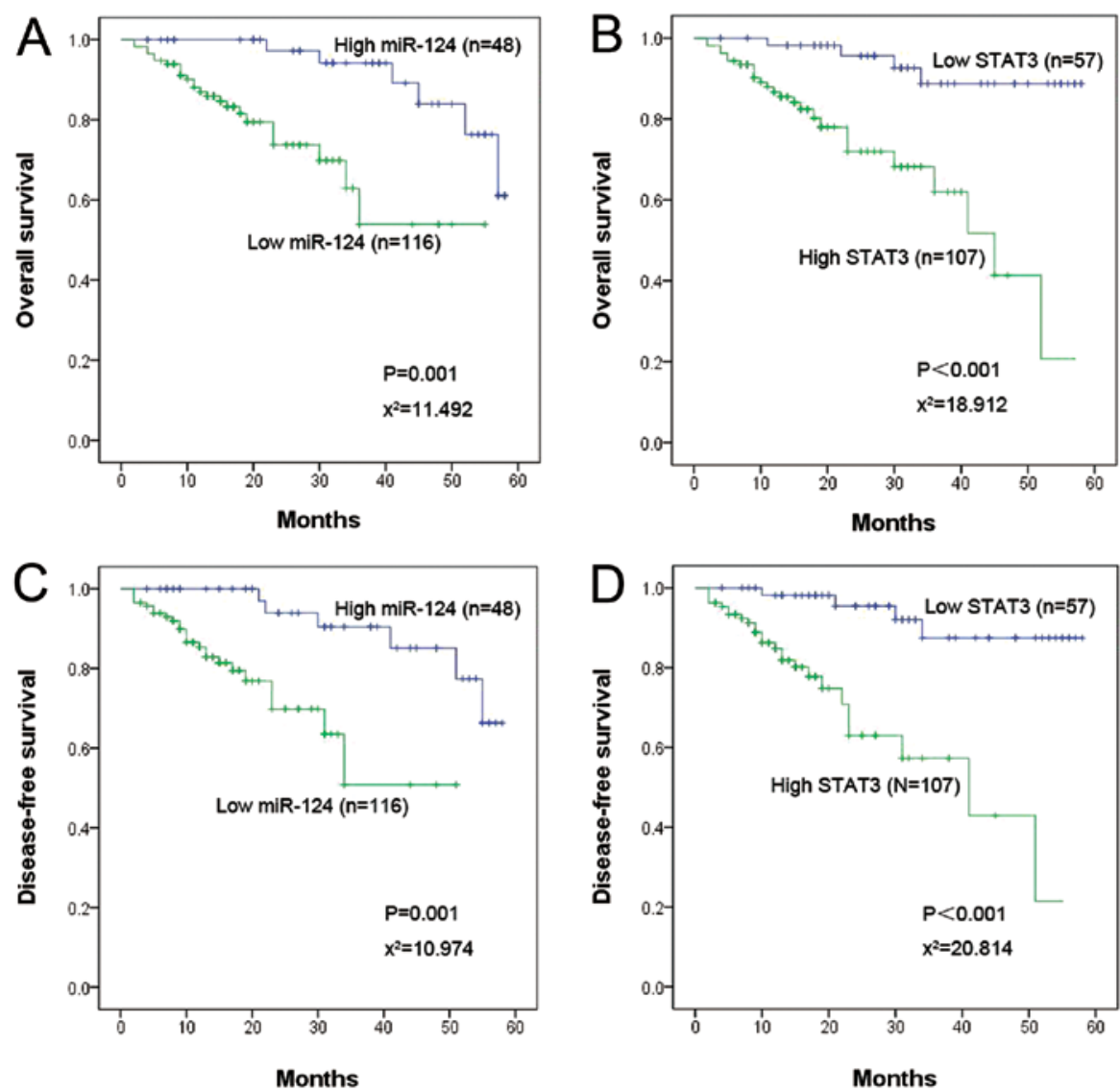

Figure 4. Kaplan-Meier survival curves for low and high miR-124 or STAT3 expression (log-rank test). (A) The expression level of miR-124 and overall survival. (B) STAT3 expression and overall survival. (C) miR-124 expression and disease-free survival. (D) STAT3 expression and disease-free survival STAT3, signal transducer and activator of transcription 3.

early or late apoptotic cells were detected in untransfected and miR-NC cells $(0.2608 \pm 0.019$ vs. $1.358 \pm 0.301 \% ; \mathrm{P}=0.059)$, whereas the cells transfected with miR-124 mimics exhibited a significantly higher percentage of early and late apoptotic cells $(20.88 \pm 2.84 ; \mathrm{P}<0.001)$ (Fig. $1 \mathrm{~F}$ and $\mathrm{G})$.

miR-124 directly targets STAT3 in NSCLC cells. STAT3 has been reported as a potential target of miR-124 during $\mathrm{T}$ cell-mediated immune clearance of glioma (16). However, the relationship between miR-124 and STAT3 has not been validated in NSCLC. We used TargetScan target prediction software (http://www.targetscan.org/) to analyze the potential interaction between miR-124 and STAT3, which is a potential target of miR-124 and contains putative miR-124 target sites in the 3' UTR (Fig. 2A). To confirm that STAT3 is a direct target of miR-124, we cloned the 3' UTR of STAT3 into the pGL3-control vector and performed reporter assays. The result showed that the relative luciferase activity of the wild-type STAT3 3' UTR reporter construct was notably decreased in A549-miR-124 cells compared to A549-miR-NC ( $\mathrm{P}=0.0024)$ or untransfected cells $(\mathrm{P}=0.0032)$. However, the relative luciferase activity of the mutant STAT3 3' UTR reporter construct was not significantly different from the luciferase activity of the A549-miR-NC cells and failed to respond to miR-124 (Fig. 2B). To further prove that STAT3 is a target of miR-124, we evaluated the expression levels of STAT3 in the three cell lines $48 \mathrm{~h}$ after transfection using real-time PCR and western blotting. The results from these assays show that STAT3 was significantly downregulated in A549-miR-124 cells compared to A549-miR-NC or untransfected cells $(\mathrm{P}<0.001)$ (Fig. 2C and D).

Expression of miR-124 and STAT3 in NSCLC tissues. miR-124 and STAT3 levels were detected in all 164 pairs of NSCLC tissues and adjacent normal lung tissue by real-time PCR. U6 snRNA, which are not deregulated in lung cancer, was used for normalization (22). These results indicated that the expression of miR-124 was significantly lower in NSCLC tissues $(0.351 \pm 0.129)$ than in adjacent normal lung tissues $(0.661 \pm 0.304 ; \mathrm{P}=0.0002)$ (Fig. 3A). The IHC analysis showed that STAT3 localized to the cytoplasm of cells (Fig. 3B). The relative expression of STAT3 was significantly higher in NSCLC tissues than adjacent normal lung tissues $(2.858 \pm 1.264$ vs. $1.476 \pm 0.672 ; \mathrm{P}<0.001$ ) (Fig. $3 \mathrm{C}$ ). In addition, Spearman's rank-order correlation analysis revealed that STAT3 expression in NSCLC tissues was inversely correlated with the expression of miR-124 ( $\left.r_{s}=-0.2642 ; P=0.0006\right)$ (Fig. 3D).

The correlation between miR-124 and STAT3 expression and clinicopathological features of NSCLC. The relationship between miR-124 or STAT3 expression and clinicopathological NSCLC characteristics is shown in Table II. The miR-124 expression levels in NSCLC tissues were closely associated with the differentiation grade $(\mathrm{P}=0.001)$, lymph node 

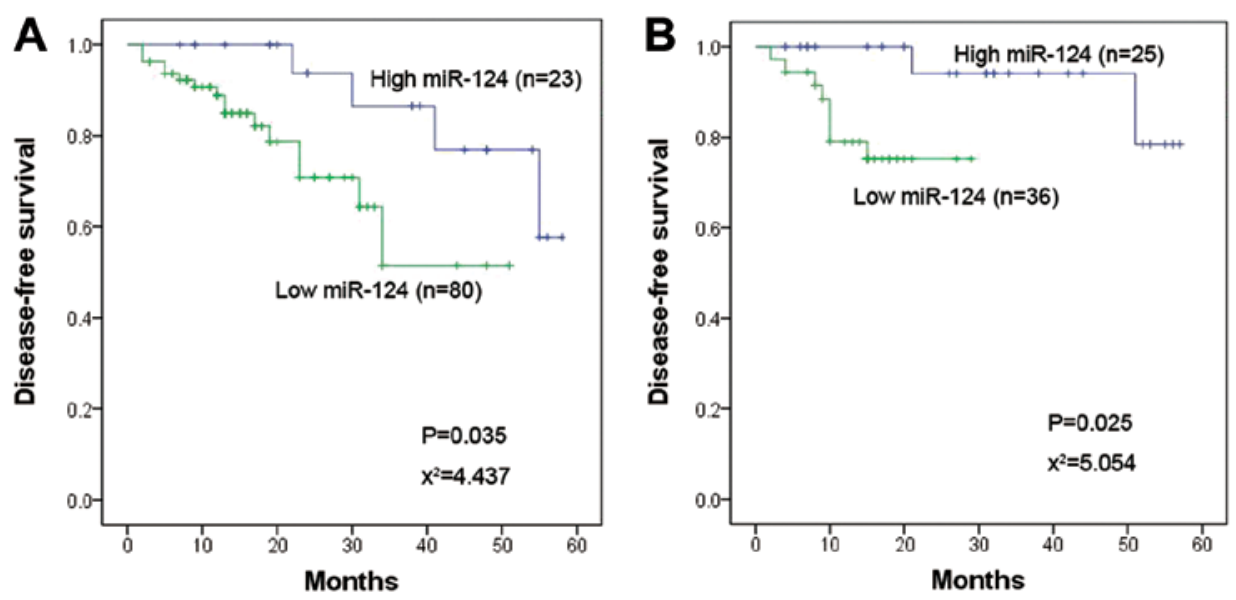

Figure 5. Kaplan-Meier survival curves for low and high miR-124 expression levels (log-rank test) in the positive and negative lymph node subgroups. (A) The expression level of miR-124 and disease-free survival in the positive lymph node subgroup. (B) The expression level of miR-124 and disease-free survival in the negative lymph node subgroup.

Table III. Cox multivariate regression analysis of miR-124 expression, STAT3 expression, gender, age, SI, tumor site, pleural invasion, pathological type, grade of differentiation and stage in relation to OS in patients with NSCLC.

\begin{tabular}{llccccc}
\hline Variables & \multicolumn{1}{c}{ Unfavorable/favorable } & $\beta$ & SE & HR & 95\% CI & P-value \\
\hline Gender & Male/female & 0.779 & 0.584 & 2.180 & $0.694-6.853$ & 0.182 \\
Age (years) & $\geq 60 /<60$ & -0.325 & 0.407 & 0.723 & $0.325-1.606$ & 0.426 \\
SI & $\geq 400 /<400$ & -0.265 & 0.539 & 0.767 & $0.267-2.205$ & 0.623 \\
Tumor site & Left/right lobe & 0.393 & 0.445 & 1.481 & $0.619-3.544$ & 0.378 \\
Pleural invasion & Present/absent & -0.463 & 0.590 & 0.502 & $0.158-1.595$ & 0.242 \\
Lymph node metastasis & Present/absent & 1.559 & 0.758 & 4.753 & $1.093-20.677$ & 0.037 \\
Pathological type & AC/SCC/others & -0.469 & 1.142 & 0.625 & $0.067-5.862$ & 0.979 \\
Differentiation & Poor/moderate/well & 0.097 & 0.836 & 1.102 & $0.214-5.670$ & 0.928 \\
Stage & IIIB/IIIA/IIB/IIA/IB/IA & 1.136 & 0.657 & 3.115 & $0.860-11.285$ & 0.161 \\
miR-124 expression & Downregulated/upregulated & -1.998 & 0.699 & 0.136 & $0.034-0.534$ & 0.004 \\
STAT3 expression & Downregulated/upregulated & -1.737 & 0.639 & 0.176 & $0.050-0.616$ & 0.007 \\
\hline
\end{tabular}

$\beta$, coefficient; SE, standard error; HR, hazard ratio; CI, confidence interval; SI, smoking index; NSCLC, non-small cell lung cancer; OS, overall survival; AC, adenocarcinoma; SCC, squamous cell carcinoma.

metastasis $(\mathrm{P}=0.017)$ and TNM stage $(\mathrm{P}=0.006)$. However, a statistical analysis revealed no significant correlations between miR-124 expression and gender, age, SI, location, pleura invasion or pathological type. As shown in Table II, STAT3 expression correlated significantly with tumor differentiation, lymph node metastasis and TNM stage $(\mathrm{P}<0.001, \mathrm{P}=0.007$ and 0.008 , respectively). In contrast, STAT3 expression did not correlate with gender, age, SI, location, pleural invasion or pathological type.

The expression of miR-124 and STAT3 and NSCLC patient prognosis. Thirty of the patients in the study died of NSCLC cancer during the follow-up period. The 1-year overall survival and DFS rates in the miR-124 upregulated group were 87.5 and $81.2 \%$, respectively, but these rates were only 68.1 and $57.8 \%$ in the miR-124 downregulated group. A KaplanMeier survival analysis was performed to further analyze the association between miR-124 and STAT3 expression and patient prognosis. The results of this analysis suggested that patients with low miR-124 or high STAT3 expression had poor OS and DFS (Fig. 4). Previously it was suggested that patients with positive lymph nodes are more prone to recurrence and metastasis compared with negative lymph node patients (23). Therefore, an additional analysis of the relationship between miR-124 expression and patient survival was performed in positive lymph node and negative lymph node patients. The results suggested that miR-124 expression was significantly associated with DFS in both positive and negative lymph node groups ( $\mathrm{P}=0.035$ vs. 0.025 , respectively) (Fig. 5A and $\mathrm{B})$. Furthermore, a Cox multivariate analysis showed that miR-124 expression (positive lymph node group, $\mathrm{P}=0.004$; negative lymph node group, $\mathrm{P}=0.009$ ), lymph node metastasis (positive lymph node group, $\mathrm{P}=0.037$; negative lymph node group, $\mathrm{P}=0.048$ ) and STAT3 expression (positive lymph node group, $\mathrm{P}=0.007$; negative lymph node group, $\mathrm{P}=0.004$ ) were significantly correlated with OS and DFS (Tables III and IV). 
Table IV. Cox multivariate regression analysis of miR-124 expression, STAT3 expression, gender, age, SI, tumor site, pleural invasion, pathological type, grade of differentiation and stage in relation to DFS in patients with NSCLC.

\begin{tabular}{llccccc}
\hline Variables & Unfavorable/favorable & $\beta$ & SE & HR & $95 \%$ CI & P-value \\
\hline Gender & Male/female & 0.706 & 0.628 & 2.026 & $0.592-6.936$ & 0.261 \\
Age (years) & $\geq 60 /<60$ & -0.265 & 0.402 & 0.767 & $0.349-1.728$ & 0.547 \\
SI & $\geq 400 /<400$ & -0.332 & 0.611 & 0.718 & $0.217-2.374$ & 0.587 \\
Tumor site & Left/right lobe & 0.492 & 0.439 & 1.635 & $0.691-3.868$ & 0.263 \\
Pleural invasion & Present/absent & -0.385 & 0.606 & 0.680 & $0.207-2.231$ & 0.525 \\
Lymph node metastasis & Present/absent & 1.535 & 0.775 & 4.640 & $1.016-21.195$ & 0.048 \\
Pathological type & AC/SCC/others & -0.182 & 1.158 & 0.833 & $0.086-8.061$ & 0.978 \\
Differentiation & Poor/moderate/well & 0.295 & 0.836 & 1.343 & $0.261-6.913$ & 0.925 \\
Stage & IIIB/IIIA/IIB/IIA/IB/IA & 1.034 & 0.671 & 2.813 & $0.756-10.471$ & 0.196 \\
miR-124 expression & Downregulated/upregulated & -1.785 & 0.686 & 0.168 & $0.044-0.644$ & 0.009 \\
STAT3 expression & Downregulated/upregulated & -1.872 & 0.648 & 0.154 & $0.043-0.548$ & 0.004 \\
\hline
\end{tabular}

$\beta$, coefficient; SE, standard error; HR, hazard ratio; CI, confidence interval; SI, smoking index; NSCLC, non-small cell lung cancer; DFS, disease-free survival; AC, adenocarcinoma; SCC, squamous cell carcinoma.

\section{Discussion}

Current research has indicated that abnormal expression of miRNAs plays a critical role in tumor formation and development $(24,25)$. miR-124 is predominantly expressed in normal brain and plays an important role in neural processes from normal neural cell function to the development of central nervous system tumors, supporting its potential role in cancer $(26,27)$. SLC16A1, the target of miR-124, regulates lactic acid export during aerobic glycolysis in medulloblastoma (28). miR-124 also inhibits the expression of SOS1 in glioblastoma (29). Recently, a number of experiments have shown that ectopic expression of miR-124 is involved in the development of other non-central nervous system tumors. For example, in hepatocellular carcinoma (HCC), cell aggressiveness is modulated by miR-124, which represses the expression of ROCK2 and EZH2 (30). In addition, in mouse models of HCC, miR-124 suppresses the expression of IL-6R and reduces STAT3 activation in transformed cells (31). In breast cancer, miR-124 inhibits the invasive and metastatic potential of cells by targeting the CD151 gene (32). Moreover, miR-124 promotes tumor progression and metastasis by downregulating Rac1 in pancreatic cancer and participates in the progression of glioblastoma by targeting PPP1R13L $(33,34)$. Taken together, these findings indicate that miR-124 is a promising tumor suppressor gene that negatively regulates oncogenes or genes that control the growth, proliferation, invasion and apoptosis of certain tumor cells by inhibiting the expression of some transcription repressors.

The results of the present study indicate that miR-124 inhibits the tumorigenic potential of lung cancer cells by downregulating STAT3. This conclusion is based on the following observations. First, overexpression of miR-124 suppressed cell proliferation and promoted apoptosis in NSCLC cells. Second, STAT3 was downregulated in NSCLC cells overexpressing miR-124 following transfection with miR-124 mimics. Third, TargetScan and luciferase activity data showed that the $3^{\prime}$ UTR of STAT3 was directly targeted by miR-124. STAT3, a member of the signal transduction and activation of transcription (STAT) family, has been shown to participate in the formation of various cancers by promoting cell proliferation, angiogenesis, and invasion or by inhibiting apoptosis $(35,36)$. In colorectal cancer, overexpression of miR-124 suppresses the growth of colorectal cancer cells by directly binding to the 3' UTR of STAT3, similar to the effects observed following knocking down of STAT3 by specific siRNA (15). As the putative primary target of miR-124, STAT3 has been studied in various cancers $(15,37,38)$. The data presented herein shed light on the relationship between miR-124 and STAT3 in lung cancer cells. In addition, we further investigated the expression of miR-124 and STAT3 at the tissue level. Our findings demonstrated that the expression of miR-124 was lower while STAT3 was higher in NSCLC tissues compared to adjacent normal lung tissues. Moreover, Spearman's rank-order correlation analysis indicated an inverse correlation between STAT3 and miR-124, further confirming that the downregulation of miR-124 resulted in STAT3 overexpression.

Accumulating studies have shown that downregulation of miR-124 is associated with a worse survival in patients with solid tumors, including pancreatic duct adenocarcinoma (33), colorectal (39) and renal cell cancer (40). The clinical data obtained in the present study revealed that a decrease in the expression of miR-124 or an increase in the expression of STAT3 correlated closely with the TNM stage, differentiation grade and lymph node metastasis. miR-124 was downregulated in patients with poor differentiation or lymph node metastasis, which suggested that its downregulation might be acquired during the process of tumor progression and perhaps even during the acquisition of metastatic potential. Berghmans et al (41) constructed a prognostic score for OS using a linear combination of miR CT values that were weighted using Cox's regression coefficients in advanced NSCLC patients treated with first-line chemotherapy. The results showed that miR-124 had no predictive role in terms of the response to chemotherapy but contributed to the prognostic outcome in OS (41). Moreover, miRNA profiling, 
which commonly included miR-124, revealed an association of miR-124 with the prognosis of stage I NSCLC after resection (42). All of these findings supported the potential prognosis value of miR-124 in NSCLC. Therefore, we further explored the relationship between the expression of miR-124 and survival in postoperative NSCLC patients. We found that a low level of miR-124 expression was associated with poor survival in terms of both DFS and OS, and a Cox proportional hazard regression analysis revealed the prognostic importance of the expression level of STAT3. Based on the above results, we concluded that miR-124 expression correlated closely with the survival of patients with NSCLC and might be a useful prognostic marker in this disease.

In conclusion, the results of the present study support the hypothesis that miR-124 is a tumor-suppressing miRNA that regulates STAT3 activity and functions as a useful biomarker for the prognosis of postoperative NSCLC patients. However, the conclusions of the present study are limited because we did not explore the detailed relationship between miR-124 and the STAT3 pathway in lung cancer cells. In addition, we did not analyze the relationship between the expression of miR-124 and adjuvant therapy. The prognostic significance of miR-124 expression in NSCLC patients receiving adjuvant therapy requires an additional prospective controlled study. Finally, additional studies in vitro and larger-scale statistical analyses of this clinical study have yet to be performed.

\section{Acknowledgements}

The present study was supported by the Jieping $\mathrm{Wu}$ Foundation of China (nos. 320.6753.1219 and 320.6750.13210), and the Department of Science and Technology of Shandong province (Contract nos. 2012YD18042, 2011YD18004 and 2010GWZ20260).

\section{References}

1. Jemal A, Bray F, Center MM, Ferlay J, Ward E and Forman D: Global cancer statistics. CA Cancer J Clin 61: 69-90, 2011.

2. Herbst RS, Heymach JV and Lippman SM: Lung cancer. N Engl J Med 359: 1367-1380, 2008.

3. Calin GA, Sevignani C, Dumitru CD, et al: Human microRNA genes are frequently located at fragile sites and genomic regions involved in cancers. Proc Natl Acad Sci USA 101: 2999-3004, 2004.

4. Johnson CD, Esquela-Kerscher A, Stefani G, et al: The let-7 microRNA represses cell proliferation pathways in human cells. Cancer Res 67: 7713-7722, 2007.

5. Kumar MS, Erkeland SJ, Pester RE, et al: Suppression of non-small cell lung tumor development by the let-7 microRNA family. Proc Natl Acad Sci USA 105: 3903-3908, 2008.

6. Takamizawa J, Konishi H, Yanagisawa K, et al: Reduced expression of the let-7 microRNAs in human lung cancers in association with shortened postoperative survival. Cancer Res 64: 3753-3756, 2004.

7. Lin PY, Yu SL and Yang PC: MicroRNA in lung cancer. Br J Cancer 103: 1144-1148, 2010.

8. Liu J, Carmell MA, Rivas FV, et al: Argonaute2 is the catalytic engine of mammalian RNAi. Science 305: 1437-1441, 2004.

9. Ambros V: The functions of animal microRNAs. Nature 431: 350-355, 2004.

10. Croce CM: Causes and consequences of microRNA dysregulation in cancer. Nat Rev Genet 10: 704-714, 2009.

11. Liu X, Sempere LF, Galimberti F, et al: Uncovering growthsuppressive MicroRNAs in lung cancer. Clin Cancer Res 15: 1177-1183, 2009
12. Ando T, Yoshida T, Enomoto S, et al: DNA methylation of microRNA genes in gastric mucosae of gastric cancer patients: its possible involvement in the formation of epigenetic field defect. Int J Cancer 124: 2367-2374, 2009.

13. Shimizu T, Suzuki H, Nojima M, et al: Methylation of a panel of microRNA genes is a novel biomarker for detection of bladder cancer. Eur Urol 63: 1091-1100, 2013.

14. Lv XB, Jiao Y, Qing Y, et al: miR-124 suppresses multiple steps of breast cancer metastasis by targeting a cohort of pro-metastatic genes in vitro. Chin J Cancer 30: 821-830, 2011.

15. Zhang J, Lu Y, Yue X, et al: MiR-124 suppresses growth of human colorectal cancer by inhibiting STAT3. PLoS One 8: e70300, 2013

16. Wei J, Wang F, Kong LY, et al: miR-124 inhibits STAT3 signaling to enhance T cell-mediated immune clearance of glioma. Cancer Res 73: 3913-3926, 2013.

17. Grivennikov SI and Karin M: Dangerous liaisons: STAT3 and NF-kappaB collaboration and crosstalk in cancer. Cytokine Growth Factor Rev 21: 11-19, 2010.

18. Zhang X, Zhang J, Wang L, Wei H and Tian Z: Therapeutic effects of STAT3 decoy oligodeoxynucleotide on human lung cancer in xenograft mice. BMC Cancer 7: 149, 2007.

19. Kanda N, Seno H, Konda Y, et al: STAT3 is constitutively activated and supports cell survival in association with survivin expression in gastric cancer cells. Oncogene 23: 4921-4929, 2004.

20. Yang G, Huang C, Cao J, Huang KJ, Jiang T and Qiu ZJ: Lentivirus-mediated shRNA interference targeting STAT3 inhibits human pancreatic cancer cell invasion. World J Gastroenterol 15: 3757-3766, 2009.

21. Ikari A, Sato T, Watanabe R, Yamazaki Y and Sugatani J: Increase in claudin-2 expression by an EGFR/MEK/ERK/c-Fos pathway in lung adenocarcinoma A549 cells. Biochim Biophys Acta 1823: 1110-1118, 2012.

22. Peltier HJ and Latham GJ: Normalization of microRNA expression levels in quantitative RT-PCR assays: identification of suitable reference RNA targets in normal and cancerous human solid tissues. RNA 14: 844-852, 2008.

23. Howington JA, Blum MG, Chang AC, Balekian AA and Murthy SC: Treatment of stage I and II non-small cell lung cancer: diagnosis and management of lung cancer, 3rd ed: American College of Chest Physicians evidence-based clinical practice guidelines. Chest 143: e278S-313S, 2013.

24. Raveche ES, Salerno E, Scaglione BJ, et al: Abnormal microRNA-16 locus with synteny to human 13q14 linked to CLL in NZB mice. Blood 109: 5079-5086, 2007.

25. Hatley ME, Patrick DM, Garcia MR, et al: Modulation of K-Rasdependent lung tumorigenesis by MicroRNA-21. Cancer Cell 18: 282-293, 2010.

26. Clark AM, Goldstein LD, Tevlin M, Tavare S, Shaham S and Miska EA: The microRNA miR-124 controls gene expression in the sensory nervous system of Caenorhabditis elegans. Nucleic Acids Res 38: 3780-3793, 2010.

27. Fowler A, Thomson D, Giles K, et al: miR-124a is frequently down-regulated in glioblastoma and is involved in migration and invasion. Eur J Cancer 47: 953-963, 2011.

28. Li KK, Pang JC, Ching AK, et al: miR-124 is frequently downregulated in medulloblastoma and is a negative regulator of SLC16A1. Hum Pathol 40: 1234-1243, 2009.

29. Lv Z and Yang L: MiR-124 inhibits the growth of glioblastoma through the downregulation of SOS1. Mol Med Rep 8: 345-349, 2013.

30. Zheng F, Liao YJ, Cai MY, et al: The putative tumour suppressor microRNA-124 modulates hepatocellular carcinoma cell aggressiveness by repressing ROCK2 and EZH2. Gut 61: 278-289, 2012.

31. Hatziapostolou M, Polytarchou C, Aggelidou E, et al: An HNF4alpha-miRNA inflammatory feedback circuit regulates hepatocellular oncogenesis. Cell 147: 1233-1247, 2011.

32. Han ZB, Yang Z, Chi Y, et al: MicroRNA-124 suppresses breast cancer cell growth and motility by targeting CD151. Cell Physiol Biochem 31: 823-832, 2013.

33. Wang P, Chen L, Zhang J, et al: Methylation-mediated silencing of the miR-124 genes facilitates pancreatic cancer progression and metastasis by targeting Rac1. Oncogene 33: 514-524, 2014.

34. Zhao WH, Wu SQ and Zhang YD: Downregulation of miR-124 promotes the growth and invasiveness of glioblastoma cells involving upregulation of PPP1R13L. Int J Mol Med 32: 101-107, 2013. 
35. Haura EB, Turkson J and Jove R: Mechanisms of disease: insight into the emerging role of signal transducers and activators of transcription in cancer. Nat Clin Pract Oncol 2: 315-324, 2005.

36. $\mathrm{Yu} \mathrm{H}$, Pardoll D and Jove R: STATs in cancer inflammation and immunity: a leading role for STAT3. Nat Rev Cancer 9: 798-809, 2009.

37. Lu Y, Yue X, Cui Y, Zhang J and Wang K: MicroRNA-124 suppresses growth of human hepatocellular carcinoma by targeting STAT3. Biochem Biophys Res Commun 441: 873-879, 2013.

38. Li Y, Zhang Z, Liu X, et al: miR-124 functions as a tumor suppressor in the endometrial carcinoma cell line HEC-1B partly by suppressing STAT3. Mol Cell Biochem 388: 219-231, 2014.

39. Wang MJ, Li Y, Wang R, et al: Downregulation of microRNA124 is an independent prognostic factor in patients with colorectal cancer. Int J Colorectal Dis 28: 183-189, 2013.
40. Gebauer K, Peters I, Dubrowinskaja N, et al: Hsa-mir-124-3 $\mathrm{CpG}$ island methylation is associated with advanced tumours and disease recurrence of patients with clear cell renal cell carcinoma. Br J Cancer 108: 131-138, 2013.

41. Berghmans T, Ameye L, Willems L, et al: Identification of microRNA-based signatures for response and survival for non-small cell lung cancer treated with cisplatin-vinorelbine $\mathrm{A}$ ELCWP prospective study. Lung Cancer 82: 340-345, 2013.

42. Patnaik SK, Kannisto E, Knudsen S and Yendamuri S: Evaluation of microRNA expression profiles that may predict recurrence of localized stage I non-small cell lung cancer after surgical resection. Cancer Res 70: 36-45, 2010. 